regurgitation in the first echocardiographic examination was found to be the only predictor of left AV valve regurgitation in the last echocardiographic examination $(p=0.001)$. There was only one patient who was reoperated for severe left AV valve regurgitation.

Conclusion The most important postoperative problem in patients with AVSD is left AV valve regurgitation. Left AV valve regurgitation in the first echocardiographic examination was found as a potential risk factor on left AV valve regurgitation.

\section{P51 UNUSUAL EARLY CLINICAL MANIFESTATION OF BLAND - WHITE - GARLAND SYNDROME IN A NEONATE}

Matija Bakoš* ${ }^{*}$ Dorotea Bartoniček, Daniel Dilber, Ivan Malčić, Dalibor Šarić. University Hospital Center, Zagreb, Croatia

10.1136/archdischild-2019-epa.406

This case report describes a 24-day-old neonate who was admitted at our hospital because of tachypnoea, tachycardia, failure to thrive, and breastfeeding fatigue.

He was a late preterm newborn; born at $36^{\text {th }}$ week of gestation with normal Apgar score. He manifested increased effort of breathing few hours after birth, while initially being treated for respiratory distress syndrome via High-Flow-NasalCannula (HFNC) with $40 \%$ of inspired oxygen supplementation for 10 days. Because of persistent tachydispnoea and feeding difficulties in maternity, hospital antimicrobial treatment was initiated unsuccessfully. Afterwards, he has been misdiagnosed with a coronary fistula and sent to our hospital. Systolic murmur was noticed. ECG showed typical changes for the left heart ischemia - anteroseptal ischemia with negative T-vawe V1-V2, aVL and DII-DIII. There were no signs of cardiogenic shock. Our ECHO revealed moderately dilated left ventricle with slightly depressed systolic function, increased echogenicity of the papillary muscles, and mild mitral regurgitation. The dilatation of right coronary artery was also shown, but the left coronary artery was not visible. The diagnosis of Bland-White-Garland syndrome was set.

The day after, he underwent cardiac catheterization when the diagnosis of anomalous origin of the left coronary artery from the pulmonary artery (ALCAPA syndrome) was confirmed. Few days later, Takeuchi procedure was performed as the method of repair. There were no major complications postoperatively. In the follow-up period he progressed favorably and now he is asymptomatic, but still has abnormal ECG. ECHO has shown significant supravalvular pulmonary stenosis.

Discussion ALCAPA is usually presented with signs of myocardial ischemia and dilated cardiomyopathy between the ages of two months throughout the adult period. The management of respiratory distress in the preterm newborn infant with oxygen supplementation had detrimental effect on myocardial perfusion and caused decrease in PVR with early presentation of DCM. This case emphasizes the importance of identifying the coronary origins as a routine part of a complete pediatric echocardiogram.

\section{P52 EVALUATION OF COMPLEMENTARY CARDIOLOGICAL TESTS IN PEDIATRIC PATIENTS WITH SYNCOPE}

Georges Akel*, Jose Siurana, Paula Ventura, Lucia Urocevich, Candela Retamozo. Hospital De Nens De Barcelona, Barcelona, Spain

10.1136/archdischild-2019-epa.407

Syncope most frequent etiopathogenic mechanism in children is Vasovagal, which is produced by decreased cerebral blood flow, and stimulation of the mechanoreceptors of the left ventricle in the context of relative central hypovolemia that causes vigorous ventricular contraction and exaggerated reflex vagal response with hypotension, bradycardia and syncope.

Objetive Analyze cardiac structure and function in pediatric patients with clinical syncope.

408 cases of patients who have consulted the Pediatric Cardiology service of the Hospital de Nens de Barcelona, from March 2013 to March 2018, with syncope, between 5 to 18 years old. To establish the discard of cardiopathy or associated vascular pathology, studies were carried out such as: electrocardiography, transthoracic echocardiography, Rhythm Holter and Ergometry with moving tape (stress test). 203 masculine, 205 female patients; single test average age: 11.2 years, 95\% Confidence Index (CI): 10.8 - 11.2. Heart rates (HR) showed progressive decrease, with greater fall between $0-7$ years, both average and minimum; mean HR: 85.34 (95\% CI: 84.2 - 86.494); and mean minimum HR: 49.1 (95\% CI: 48.2 - 49.9). In the Pearson Correlation Analysis, negative results were evidenced for mean and minimum $\mathrm{HR}$, for age: maximum HR: -0.291'; average HR: -0.586' and minimum HR: 0.452'; and sex: maximum HR: 0.022; average HR: 0.129' and minimum: 0.0184'. In Rhythm Holter: 79.7\% of patients $(n=325)$ had normal results; $6.1 \% \quad(n=25)$ : second-degree atrioventricular block type $1 ; 5.4 \% \quad(n=22)$ supraventricular extrasystoles; $4.4 \% \quad(n=18)$ ventricular extrasystoles; $2.5 \% \quad(\mathrm{n}=10)$ first degree atrioventricular block; $0.5 \%(n=2)$ sinus bradycardia; $0.5 \%(n=2)$ second degree atrioventricular block type 2;0.4\% $(n=2)$ Wolf Parkinson's White syndrome. All patients had normal anatomical and functional cardiac evaluations, except 1 patient $(0.2 \%)$ : severe systolic and diastolic dysfunction FE: 49\%, second-degree auto-ventricular block type 2 II grade 2, and highly impaired ergometry: HR under maximum: 50\%, syncope with any movement. Ergometry: 406 patients obtained normal results, maximum heart rates - $87.7 \%$ average of $100 \%$ calculated per patient (95\% CI 87.2 . 88.3). Mets obtained - single test average: 13.7 IC (13.4 14.0). 2 patients with less than $80 \%$ maximum heart rate, due to dyspnea due to physical exertion, presented normal echocardiography and altered rhythm Holter with diagnoses of second degree atrioventricular block type II.

Conclusions Most of the patients analyzed with rhythm Holter, echocardiography and ergometry, did not present diagnoses related to syncopal symptoms. Only 1 patient with syncope $(0.2 \%)$ : severe diastolic and systolic dysfunction, second-degree atrioventricular block type 2 II grade 2, and highly altered ergometry. 